\title{
Multiphase Segmentation of Deformation using Logarithmic Priors
}

\author{
Igor Yanovsky $^{1} \quad$ Paul M. Thompson ${ }^{2} \quad$ Stanley Osher $^{1} \quad$ Luminita Vese $^{1} \quad$ Alex D. Leow ${ }^{2}$ \\ ${ }^{1}$ Department of Mathematics, University of California, Los Angeles, CA 90095 \\ ${ }^{2}$ Laboratory of Neuro Imaging, UCLA School of Medicine, Los Angeles, CA 90095
}

\begin{abstract}
In [8], the authors proposed the large deformation logunbiased diffeomorphic nonlinear image registration model which has been successfully used to obtain theoretically and intuitively correct deformation maps. In this paper, we extend this idea to simultaneously registering and tracking deforming objects in a sequence of two or more images. We generalize a level set based Chan-Vese multiphase segmentation model to consider Jacobian fields while segmenting regions of growth and shrinkage in deformations. Deforming objects are thus classified based on magnitude of homogeneous deformation. Numerical experiments demonstrating our results include a pair of two-dimensional synthetic images and pairs of two-dimensional and three-dimensional serial MRI images.
\end{abstract}

\section{Introduction}

Segmentation of homogeneous deformation is a challenging problem which incorporates several image processing and computer vision areas including image registration, segmentation, and tracking. The goal of deformation segmentation is to classify regions of homogeneous volume/density change based on magnitude of such change. In this work, we employ a robust image registration model for generating priors for further segmentation of deformed features.

Image registration models are used to align, or spatially normalize, one image to match another. Presented with a choice of an image registration method, it is important to ensure that the model in consideration generates meaningful deformation fields. In general, the transformation that defines the correspondence map between the images should be diffeomorphic, to preserve the topology. As was described in [8], not all widely used image registration models generate theoretically and intuitively correct deformation fields. In [8], the authors introduced the information theory approach to quantifying deformation, proposing a framework for constructing large deformation diffeomorphic im-

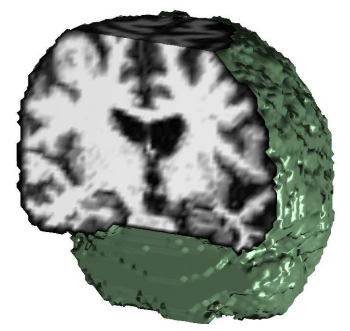

$T$

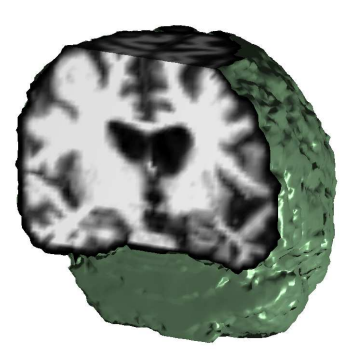

$T \circ \vec{h}$
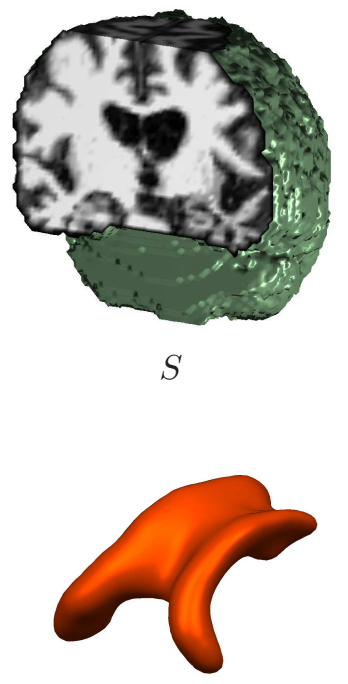

segmentation of deformed region
Figure 1. Segmentation of deformation for 3D serial MRI image. Volume cuts of image $T$, image $S$, and deformed image $T$ are shown. The surface (zero level set of function $\phi$ ) of the ventricle is shown. The ventricle surface is enlarged for better visualization.

age registration models. Section 2 of this paper describes how to employ logarithmic priors in order to generate theoretically and intuitively correct deformation maps.

Even though a robust nonlinear registration model can generate meaningful deformation maps, an automated segmentation process would be required to classify regions of homogeneous deformation in a sequence of images. In Section 3 we generalize level set based active contour without edges model [2] to classify regions of homogeneous deformation.

\section{Log-Unbiased Image Registration}

Let us denote the template image as $T(\vec{x})$ and the study image as $S(\vec{x})$, which are images defined on the spatial 
domain $\Omega$. The problem of image registration is to find a smooth deformation $\vec{h}$, such that the deformed template $T \circ \vec{h}(\vec{x})$ is in some sense close to $S(\vec{x})$. The deformation $\vec{h}$ is usually expressed at each voxel in terms of the displacement vector $\vec{u}$ from the original position: $\vec{h}(\vec{x})=\vec{x}-\vec{u}(\vec{x})$. It is desirable to obtain a bijective deformation $\vec{h}$ that is diffeomorphic and topology preserving. The inverse map of $\vec{h}$ is denoted as $\vec{h}^{-1}$ and the Jacobian matrix of $\vec{h}$ as $D \vec{h}$. The Jacobian map can thus be defined as the determinant of the Jacobian matrix $|D \vec{h}|$.

In [8], the authors proposed to minimize the following energy functional:

$$
\begin{gathered}
E(T, S, \vec{u})=\frac{1}{2} \int_{\Omega}|T(\vec{x}-\vec{u})-S(\vec{x})|^{2} d \vec{x} \\
+\lambda \int_{\Omega}(|D \vec{h}(\vec{x})|-1) \log |D \vec{h}(\vec{x})| d \vec{x},
\end{gathered}
$$

where $\lambda>0$ is the Lagrange multiplier. The first term of the energy functional in (1), referred to as the sum of the square differences (SSD), forces the deformed template to match the study. The integrand of the second term is always non-negative, and only evaluates to zero when $\vec{h}$ is volumepreserving everywhere $(|D \vec{h}| \equiv 1)$. Thus, minimizing the second term leads to unbiased deformation in the logarithmic space, ensuring the deformation be diffeomorphic.

The functional in (1) is minimized using the gradient descent of the corresponding Euler-Lagrange equations to obtain the force field (or body force) $\vec{f}$, which drives the template into registration with the study:

$$
\vec{f}(\vec{x}, \vec{u}(\vec{x}, t))=-\frac{\partial E(T, S, \vec{u})}{\partial \vec{u}} .
$$

Here, $t$ is an artificial time.

We solve the viscous fluid model proposed by Christensen et al. [3]. Of note, in [3], the authors used the SSD as a cost functional for minimization (no control over the distribution of the Jacobian values was employed). Given the velocity field $\vec{v}$, the following partial differential equation can be solved to obtain the displacement field $\vec{u}$ :

$$
\frac{\partial \vec{u}}{\partial t}=\vec{v}-\vec{v} \cdot \vec{\nabla} \vec{u} .
$$

The instantaneous velocity as in [4] is obtained by convolving $\vec{f}$ with Gaussian kernel $G_{\sigma}$ of variance $\sigma$ :

$$
\vec{v}=G_{\sigma} *(-\vec{f}(\vec{x}, \vec{u})) .
$$

We solve this equation using the Fast Fourier transform (FFT).

\section{Segmentation of Deforming Objects}

The unbiased registration model described in Section 2 yields theoretically and intuitively correct deformation maps $\vec{h}$. Jacobian maps of such deformations closely depict the underlying volume/density changes the modeled systems undergo. In this section, we describe the two-phase segmentation model, originally used for segmenting images based on their intensity values, and explain how the model can be generalized to classify the regions of homogeneous deformation obtained with the unbiased registration model.

\subsection{The Chan-Vese Intensity Based Segmentation Model}

The One Level Set (Two-Phase) Framework. Based on the Mumford and Shah functional [5] for segmentation, Chan and Vese [2] proposed a level set method based active contour model to detect objects whose boundaries are not necessarily defined by a gradient.

Let us denote a given image by $I_{0}: \Omega \rightarrow \mathbb{R}$ and suppose $C(C=\partial R)$ is a hypersurface representing a boundary of a region of interest $R \subset \Omega$. The Chan-Vese (CV) model minimizes the following energy:

$$
\begin{gathered}
F_{2}^{C V}\left(c_{1}, c_{2}, C\right)=\int_{R_{1}=R}\left(I_{0}(\vec{x})-c_{1}\right)^{2} d \vec{x} \\
+\int_{R_{2}=\Omega \backslash R}\left(I_{0}(\vec{x})-c_{2}\right)^{2} d \vec{x}+\beta \int_{\partial R} d s,
\end{gathered}
$$

where $c_{1}, c_{2}$ are unknown constants, and $\beta>0$ is the length parameter. This problem is solved using the level set method of Osher and Sethian [6]. In a level set formulation, a hypersurface $C$ is represented implicitly by the zero level set of a Lipshitz continuous function $\phi: \Omega \rightarrow \mathbb{R}$, such that:

$$
\phi(\vec{x})<0 \text { in } R, \quad \phi(\vec{x})>0 \text { in } \Omega \backslash R .
$$

The Chan-Vese functional in (5) written in the level set formulation is:

$$
\begin{aligned}
& F_{2}^{C V}\left(c_{1}, c_{2}, \phi\right)=\int_{\Omega}\left(I_{0}(\vec{x})-c_{1}\right)^{2}(1-H(\phi)) d \vec{x} \\
& +\int_{\Omega}\left(I_{0}(\vec{x})-c_{2}\right)^{2} H(\phi) d \vec{x}+\beta \int_{\Omega}|\nabla H(\phi)| d \vec{x},
\end{aligned}
$$

where $H(y)$ is a heaviside function. The functional is minimized using incremental updating along the gradient descent direction of the Euler-Lagrange equation in $\phi$ :

$$
\begin{aligned}
\frac{\partial \phi}{\partial t} & =\delta(\phi)\left[\beta \nabla \cdot\left(\frac{\nabla \phi}{|\nabla \phi|}\right)\right. \\
& \left.+\left(I_{0}-c_{1}\right)^{2}-\left(I_{0}-c_{2}\right)^{2}\right],
\end{aligned}
$$

where $\delta(y)$ is the delta function and $t$ is an artificial time. The constants $c_{1}$ and $c_{2}$ are evaluated as

$$
\begin{aligned}
& c_{1}(\phi)=\frac{\int_{\Omega} I_{0}(\vec{x})(1-H(\phi(t, \vec{x}))) d \vec{x}}{\int_{\Omega}(1-H(\phi(t, \vec{x}))) d \vec{x}}, \\
& c_{2}(\phi)=\frac{\int_{\Omega} I_{0}(\vec{x}) H(\phi(t, \vec{x})) d \vec{x}}{\int_{\Omega} H(\phi(t, \vec{x})) d \vec{x}} .
\end{aligned}
$$




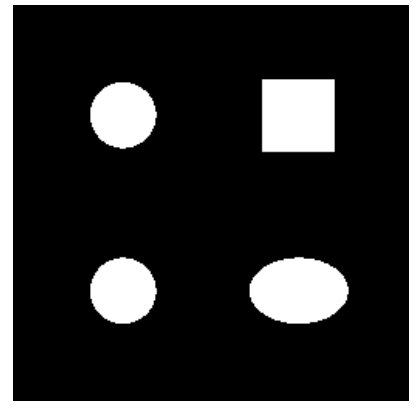

(a) $T$

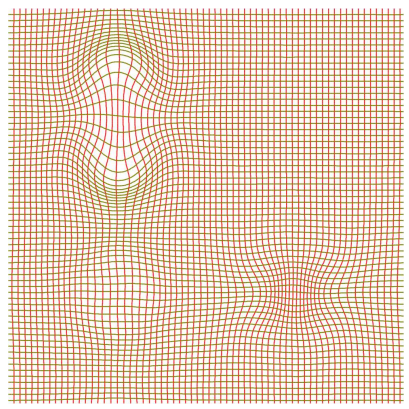

(e) deformed grid

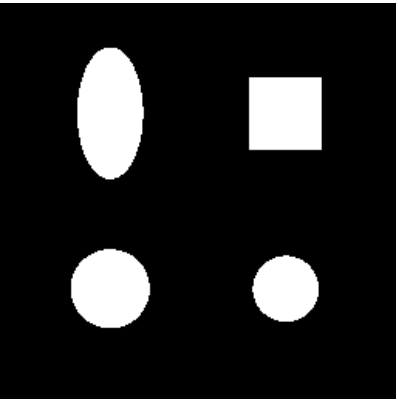

(b) $S$

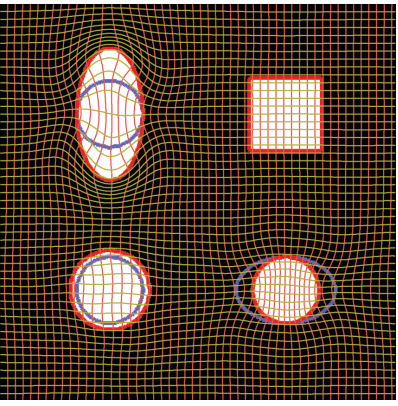

(f) $T \circ \vec{h}$ and grid

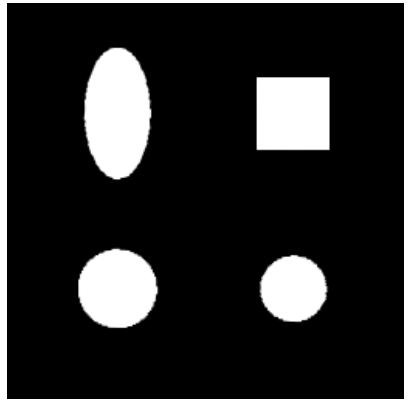

(c) $T \circ \vec{h}$

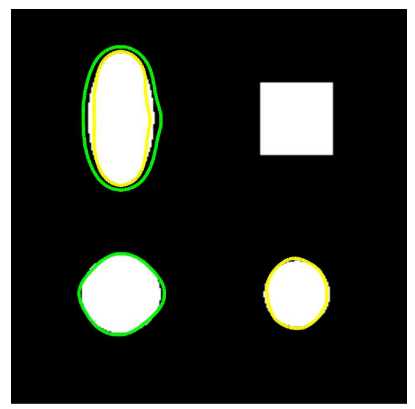

(g) segmentation contours

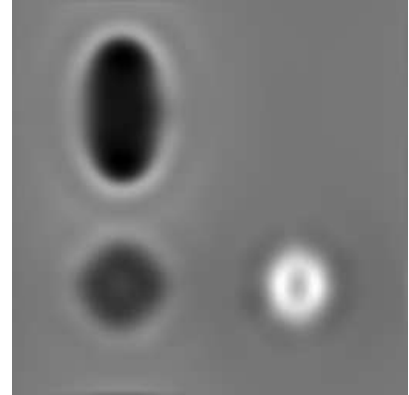

(d) $|D \vec{h}|$

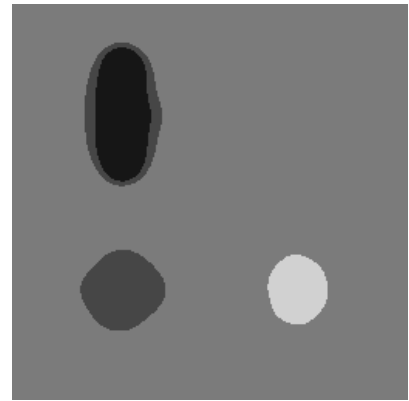

(h) segmentation of $|D \vec{h}|$

Figure 2. Segmentation of deformation for a synthetic image. (a) image $T$; (b) image $S$; (c) image $T$ is deformed into image $S$. The upperleft circle in the image undergoes the largest expansion (density change), while the lower-right ellipsoid undergoes the contraction. The upper-right square does not deform. (d) The Jacobian map of the deformation. Dark and bright spots represent expanding and contracting areas, respectively. (e) The deformed grid; (f) the deformed grid and the deformed image. Here, blue, yellow, and red contours represent boundaries of objects in $T, S$, and deformed $T$, respectively. (g) Segmentation results are obtained using the four-phase (multiphase) segmentation model, which enables to find up to four regions in the image. Green and yellow contours represent the zero level sets of $\phi_{1}$ and $\phi_{2}$, respectively. The four regions of homogeneous change in density are located. (h) The segmented Jacobian map is displayed.

Note that $c_{1}$ and $c_{2}$ are the averages of the intensities of $I_{0}$ inside and outside $C$, respectively. The twophase segmentation of the image $I_{0}(\vec{x})$ is given by $I(\vec{x})=c_{1}(1-H(\phi(\vec{x})))+c_{2} H(\phi(\vec{x}))$.

The Two Level Set (Multiphase) Framework. In [7], the authors generalized the one level set active contour without edges model to two or more level set multiphase framework. A four-phase model, described in this section, allows for up to four regions to be segmented. Here, we suppose $C_{1}$ and $C_{2}$ are hypersurfaces separating an image into four disjoint regions $R_{i} \subset \Omega, 1 \leq i \leq 4$. Some of these regions are allowed to be empty. The four phase model thus minimizes the following energy:

$$
\begin{aligned}
& F_{4}^{C V}\left(c_{11}, c_{12}, c_{21}, c_{22}, C_{1}, C_{2}\right) \\
& =\int_{R_{1}}\left(I_{0}(\vec{x})-c_{11}\right)^{2} d \vec{x}+\int_{R_{2}}\left(I_{0}(\vec{x})-c_{12}\right)^{2} d \vec{x} \\
& \quad+\int_{R_{3}}\left(I_{0}(\vec{x})-c_{21}\right)^{2} d \vec{x}+\int_{R_{4}}\left(I_{0}(\vec{x})-c_{22}\right)^{2} d \vec{x} \\
& \quad+\beta \int_{C_{1}} d s+\beta \int_{C_{2}} d s,
\end{aligned}
$$

where $c_{11}, c_{12}, c_{21}, c_{22}$ are unknown constants, and $\beta>0$ is the length parameter. Representing $C_{1}$ and $C_{2}$ implicity as zero level sets of functions $\phi_{1}$ and $\phi_{2}$, respectively, such that

$$
\begin{array}{ll}
\phi_{1}(\vec{x})<0, \phi_{2}(\vec{x})<0 & \text { in } R_{1}, \\
\phi_{1}(\vec{x})<0, \phi_{2}(\vec{x})>0 & \text { in } R_{2}, \\
\phi_{1}(\vec{x})>0, \phi_{2}(\vec{x})<0 & \text { in } R_{3}, \\
\phi_{1}(\vec{x})>0, \phi_{2}(\vec{x})>0 & \text { in } R_{4},
\end{array}
$$

we can write the Chan-Vese functional in (10) in the level set formulation as:

$$
\begin{aligned}
& F_{4}^{C V}\left(c_{11}, c_{12}, c_{21}, c_{22}, \phi_{1}, \phi_{2}\right) \\
& =\int_{\Omega}\left(I_{0}(\vec{x})-c_{11}\right)^{2}\left(1-H\left(\phi_{1}\right)\right)\left(1-H\left(\phi_{2}\right)\right) d \vec{x} \\
& +\int_{\Omega}\left(I_{0}(\vec{x})-c_{12}\right)^{2}\left(1-H\left(\phi_{1}\right)\right) H\left(\phi_{2}\right) d \vec{x} \\
& +\int_{\Omega}\left(I_{0}(\vec{x})-c_{21}\right)^{2} H\left(\phi_{1}\right)\left(1-H\left(\phi_{2}\right)\right) d \vec{x} \\
& +\int_{\Omega}\left(I_{0}(\vec{x})-c_{22}\right)^{2} H\left(\phi_{1}\right) H\left(\phi_{2}\right) d \vec{x} \\
& +\beta \int_{\Omega}\left|\nabla H\left(\phi_{1}\right)\right| d \vec{x}+\beta \int_{\Omega}\left|\nabla H\left(\phi_{2}\right)\right| d \vec{x} .
\end{aligned}
$$

This functional can be minimized using the gradient descent of the corresponding Euler-Lagrange equations for $\phi_{1}$ and 


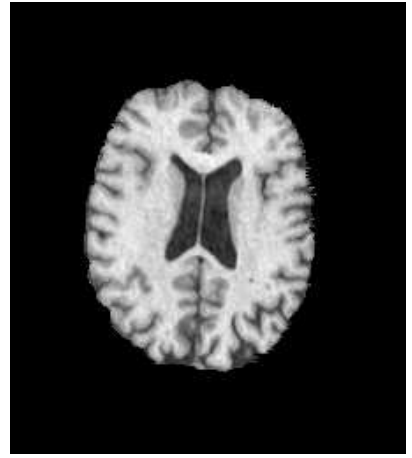

(a) $T$

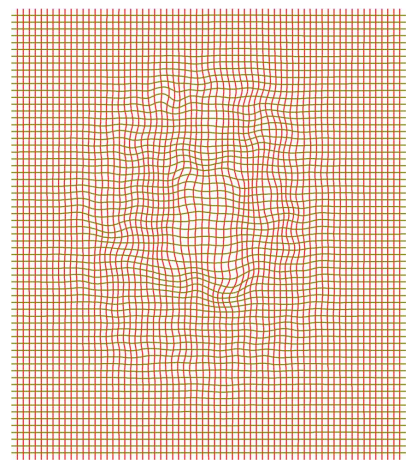

(e) deformed grid

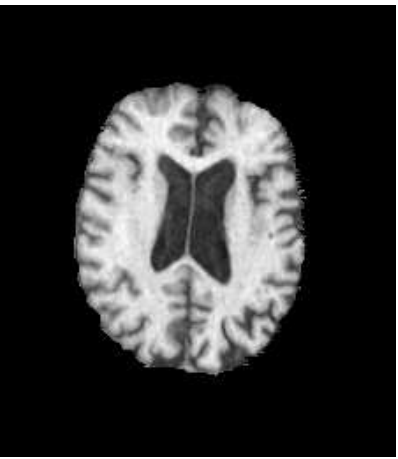

(b) $S$

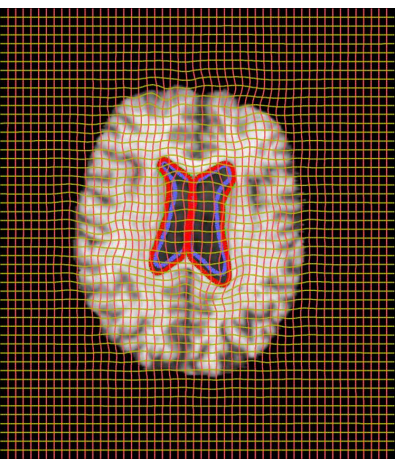

(f) $T \circ \vec{h}$ and grid

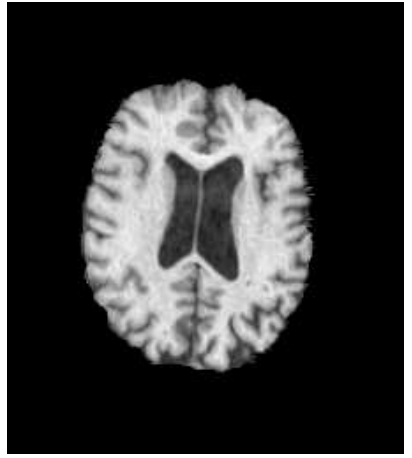

(c) $T \circ \vec{h}$

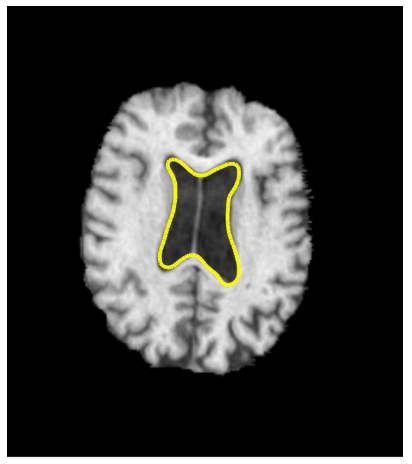

(g) segmentation contours

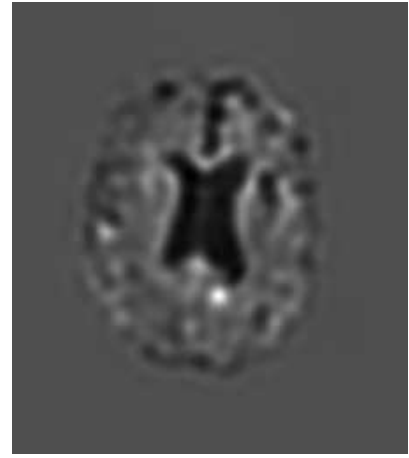

(d) $|D \vec{h}|$

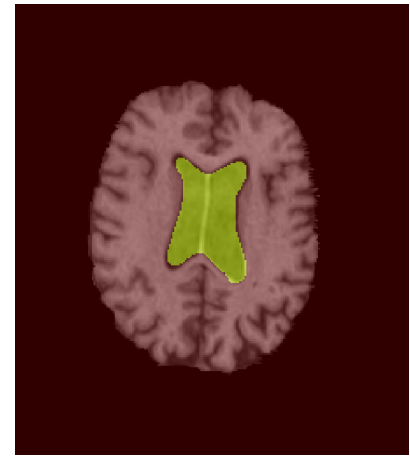

(h) $T \circ \vec{h}$ and segmentation

Figure 3. Segmentation of deformation for 2D serial MRI image. (a) image $T$; (b) image $S$; (c) image $T$ is deformed into image $S$. The ventricle is noticeably enlarged. (d) The Jacobian map of the deformation; (e) the deformed grid; (f) the deformed grid and the deformed image. Here, blue, yellow, and red contours represent the boundaries of objects in $T, S$, and deformed $T$, respectively. (g) Segmentation results are obtained using the four-phase (multiphase) segmentation model, which enables to find up to four regions in the image. However, since only the ventricle had undergone the deformation, the image is partitioned into two parts. (h) The deformed image is superimposed with the segmentation of the deformation.

$\phi_{2}$ to obtain the following evolution equations:

$$
\begin{aligned}
\frac{\partial \phi_{1}}{\partial t} & =\delta\left(\phi_{1}\right)\left[\beta \nabla \cdot\left(\frac{\nabla \phi_{1}}{\left|\nabla \phi_{1}\right|}\right)\right. \\
& +\left(\left(I_{0}-c_{11}\right)^{2}-\left(I_{0}-c_{21}\right)^{2}\right)\left(1-H\left(\phi_{2}\right)\right) \\
& \left.+\left(\left(I_{0}-c_{12}\right)^{2}-\left(I_{0}-c_{22}\right)^{2}\right) H\left(\phi_{2}\right)\right], \\
\frac{\partial \phi_{2}}{\partial t} & =\delta\left(\phi_{2}\right)\left[\beta \nabla \cdot\left(\frac{\nabla \phi_{2}}{\left|\nabla \phi_{2}\right|}\right)\right. \\
& +\left(\left(I_{0}-c_{11}\right)^{2}-\left(I_{0}-c_{12}\right)^{2}\right)\left(1-H\left(\phi_{1}\right)\right) \\
& \left.+\left(\left(I_{0}-c_{21}\right)^{2}-\left(I_{0}-c_{22}\right)^{2}\right) H\left(\phi_{1}\right)\right] .
\end{aligned}
$$

The constants $c_{11}, c_{12}, c_{21}, c_{22}$ are evaluated as

$$
\begin{aligned}
c_{11}\left(\phi_{1}, \phi_{2}\right) & =\frac{\int_{\Omega} I_{0}\left(1-H\left(\phi_{1}\right)\right)\left(1-H\left(\phi_{2}\right)\right) d \vec{x}}{\int_{\Omega}\left(1-H\left(\phi_{1}\right)\right)\left(1-H\left(\phi_{2}\right)\right) d \vec{x}}, \\
c_{12}\left(\phi_{1}, \phi_{2}\right) & =\frac{\int_{\Omega} I_{0}\left(1-H\left(\phi_{1}\right)\right) H\left(\phi_{2}\right) d \vec{x}}{\int_{\Omega}\left(1-H\left(\phi_{1}\right)\right) H\left(\phi_{2}\right) d \vec{x}}, \\
c_{21}\left(\phi_{1}, \phi_{2}\right) & =\frac{\int_{\Omega} I_{0} H\left(\phi_{1}\right)\left(1-H\left(\phi_{2}\right)\right) d \vec{x}}{\int_{\Omega} H\left(\phi_{1}\right)\left(1-H\left(\phi_{2}\right)\right) d \vec{x}}, \\
c_{22}\left(\phi_{1}, \phi_{2}\right) & =\frac{\int_{\Omega} I_{0} H\left(\phi_{1}\right) H\left(\phi_{2}\right) d \vec{x}}{\int_{\Omega} H\left(\phi_{1}\right) H\left(\phi_{2}\right) d \vec{x}} .
\end{aligned}
$$

Here, $c_{11}, c_{12}, c_{21}$, and $c_{22}$ correspond to averages of intensities of $I_{0}$ in $R_{1}, R_{2}, R_{3}$, and $R_{4}$, respectively. The four-phase segmentation of the image $I_{0}(\vec{x})$ is given by

$$
\begin{aligned}
I(\vec{x}) & =c_{11}\left(1-H\left(\phi_{1}\right)\right)\left(1-H\left(\phi_{2}\right)\right) \\
& +c_{12}\left(1-H\left(\phi_{1}\right)\right) H\left(\phi_{2}\right) \\
& +c_{21} H\left(\phi_{1}\right)\left(1-H\left(\phi_{2}\right)\right) \\
& +c_{22} H\left(\phi_{1}\right) H\left(\phi_{2}\right) .
\end{aligned}
$$

\subsection{Jacobian Based Segmentation using the CV Model}

Instead of segmenting the image based on its intensity values, we propose to classify and track regions of homogeneous deformation using the Jacobian values $|D \vec{h}|$. As a result, the two-phase Chan-Vese functional in (7) applied to segmentation of deformation becomes

$$
\begin{aligned}
& F_{2}\left(c_{1}, c_{2}, \phi\right)=\int_{\Omega}\left(|D \vec{h}(\vec{x})|-c_{1}\right)^{2}(1-H(\phi)) d \vec{x} \\
& +\int_{\Omega}\left(|D \vec{h}(\vec{x})|-c_{2}\right)^{2} H(\phi) d \vec{x}+\beta \int_{\Omega}|\nabla H(\phi)| d \vec{x},
\end{aligned}
$$


The multiphase functional in (12) for homogeneous deformation segmentation becomes

$$
\begin{aligned}
& F_{4}\left(c_{11}, c_{12}, c_{21}, c_{22}, \phi_{1}, \phi_{2}\right) \\
& =\int_{\Omega}\left(|D \vec{h}(\vec{x})|-c_{11}\right)^{2}\left(1-H\left(\phi_{1}\right)\right)\left(1-H\left(\phi_{2}\right)\right) d \vec{x} \\
& +\int_{\Omega}\left(|D \vec{h}(\vec{x})|-c_{12}\right)^{2}\left(1-H\left(\phi_{1}\right)\right) H\left(\phi_{2}\right) d \vec{x} \\
& +\int_{\Omega}\left(|D \vec{h}(\vec{x})|-c_{21}\right)^{2} H\left(\phi_{1}\right)\left(1-H\left(\phi_{2}\right)\right) d \vec{x} \\
& +\int_{\Omega}\left(|D \vec{h}(\vec{x})|-c_{22}\right)^{2} H\left(\phi_{1}\right) H\left(\phi_{2}\right) d \vec{x} \\
& +\beta \int_{\Omega}\left|\nabla H\left(\phi_{1}\right)\right| d \vec{x}+\beta \int_{\Omega}\left|\nabla H\left(\phi_{2}\right)\right| d \vec{x},
\end{aligned}
$$

with the corresponding generalizations of equations (13) and (14).

Of note, for providing additional flexibility, both intensity values and the Jacobian field could be incorporated as two channels into a multichannel model described in [1]. Depending on an application, additional channels may be incorporated into the model.

\section{Results}

In this section, we tested the proposed segmentation of homogeneous deformation framework. In the first numerical example in Figure 2 we considered matching two synthetic images (each of size 256 by $256, \lambda=1000$ in (1)). The geometrical objects on each of these images (Figure 2(a,b)) are of identical intensity; however, each of these four objects undergoes a deformation of a different magnitude (Figure 2(d,e,f)). The upper-left object (a circle to an ellipse transformation) undergoes the biggest positive deformation (expansion) and the lower-right object (an ellipse to a circle transformation) is being contracted. Note that the square does not deform. In this example, the segmentation was done using the four-phase segmentation model (17) with the length parameter $\beta=0.02 \cdot 255^{2}$. The four regions of homogeneous deformation were detected (Figure 2(g,h)). The background and non-deforming square were classified as a single region of zero (or almost zero) deformation.

In Figure 3, we show the results of matching a pair of 2D slices (Figure 3(a,b)) from a set of serial MRI images (each of size 226 by $256, \lambda=400$ in (1)), where visually significant ventricle enlargement is present. Here, it is desirable to distinguish the region of ventricular expansion from the rest of the image. This is successfully accomplished using the segmentation of homogeneous deformation procedure (Figure 3(g,h)). The four-phase segmentation model was employed in this example (with the length parameter $\beta=0.1 \cdot 255^{2}$ in (17)), locating only two regions of homogeneous deformation, which is intuitively correct.

In the last numerical example (Figures 1 and 4), we tested the proposed model using a pair of 3D serial MRI vol-
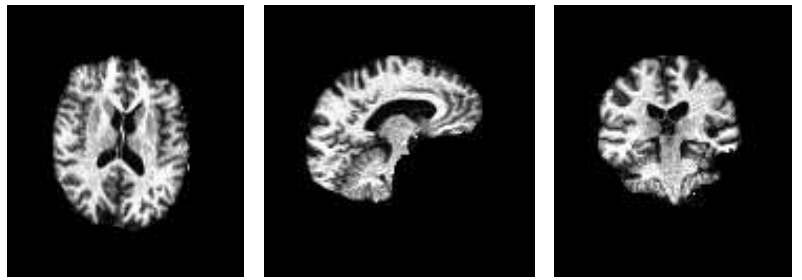

$T$
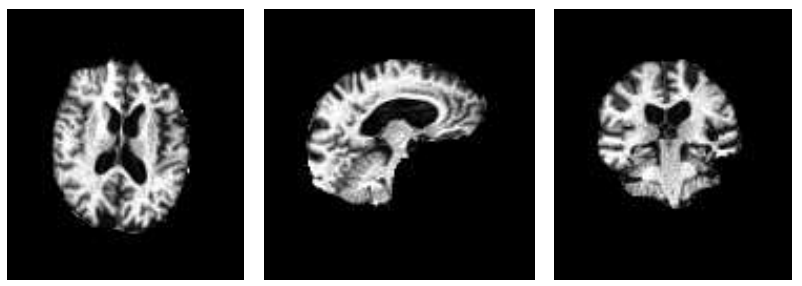

$S$
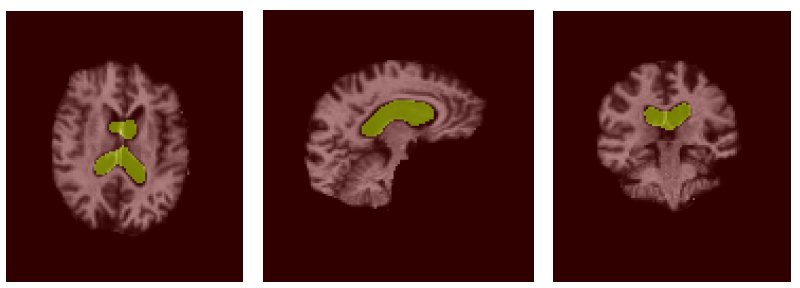

$T \circ \vec{h}$ and segmentation

Figure 4. Segmentation of deformation for 3D serial MRI image. Columns depict: axial (column 1), sagittal (column 2), and coronal (column 3 ) slices of image $T$; image $S$; deformed image $T$ superimposed with the segmentation of deformation. Segmentation results are obtained using the two-phase segmentation model, which enables to separate two regions in the image. Since the ventricle underwent the largest deformation, it is separated from the rest of the image.

umes (each of size $112 \times 128 \times 128$ ) which, similar to a previous $2 \mathrm{D}$ example, display significant ventricular growth. A fully three-dimensional computation was employed, with $\lambda=500$ and $\beta=0.05 \cdot 255^{2}$ in a two-phase segmentation model (16). Figure 1 displays the volume cuts of the two volumes matched as well as the result of segmentation in the form of a surface (zero level set of function $\phi$ ) of the ventricle. The two-dimensional slices of the $3 \mathrm{D}$ volume, as well as the corresponding segmentation of deformation, are shown in Figure 4. The region of growth was identified and separated from the rest of the image in this example.

\section{Acknowledgements}

This work was supported by Grants U54 RR021813 NIH/NCRR, Grant U01 AG024904, and Grants R21 RR019771, EB01651, AG016570, NS049194 to PT.

The authors would like to thank James Becker and Simon Davis at the University of Pittsburgh for providing the MRI dataset. 


\section{References}

[1] T. F. Chan, B. Y. Sandberg, and L. A. Vese. Active contours without edges for vector-valued images. J. of Visual Comm. and Image Rep., 11(2):130-141, 2000.

[2] T. F. Chan and L. A. Vese. Active contours without edges. IEEE Transactions on Image Processing, 10(2):266-277, 2001.

[3] G. Christensen, R. Rabbitt, and M. Miller. Deformable templates using large deformation kinematics. IEEE Transactions on Image Processing, 5(10):1435-1447, 1996.

[4] E. D'Agostino, F. Maes, D. Vandermeulen, and P. Suetens. A viscous fluid model for multimodal non-rigid image registration using mutual information. Medical Image Analysis, 7:565-575, 2003.

[5] D. Mumford and J. Shah. Optimal approximations by piecewise smooth functions and associated variational problems. Commun. Pure Appl. Math., 42:577-685, 1989.

[6] S. Osher and J. Sethian. Fronts propagating with curvature dependent speed; algorithms based on Hamilton-Jacobi formulations. J. Comput. Phys., 79:12-49, 1988.

[7] L. Vese and T. Chan. A multiphase level set framework for image segmentation using the Mumford and Shah model. International Journal of Computer Vision, 50(3):271-293, 2002.

[8] I. Yanovsky, P. Thompson, S. Osher, and A. Leow. Topology preserving log-unbiased nonlinear image registration: Theory and implementation. IEEE Conference on Computer Vision and Pattern Recognition, 2007. 